\title{
Intraoperative Switch to a Temporal Surgical Approach in 23- and 25-Gauge Microcannula-Based Sutureless Transconjunctival Vitrectomy
}

\author{
Szilárd Kiss ${ }^{1,2}$ and Demetrios Vavvas ${ }^{*}, 2$ \\ ${ }^{I}$ Retina Service, Weill Cornell Medical College, Department of Ophthalmology, 1305 York Ave, $11^{\text {th }}$ Floor, New York, \\ New York 10021, USA \\ ${ }^{2}$ Retina Service, Massachusetts Eye and Ear Infirmary, Harvard Medical School Department of Ophthalmology, 243 \\ Charles St., Boston, Massachusetts 02114, USA
}

\begin{abstract}
Purpose: To illustrate a surgical method in which the infusion port during a three-port pars plana vitrectomy is moved intraoperatively from the traditional infra-temporal location and placed supra-nasally, thus permitting a temporal surgical approach to better tackle superior and inferior vitreoretinal pathology.

Methods: Description of surgical technique.

Results: When the location of the vitreoretinal pathology and/or the patient's anatomy prevents adequate visualization or surgical access and/or the instrument flexibility precludes sufficient maneuvering of the eyeball, a temporal approach to the vitrectomy may be employed by utilizing the interchangeable microcannulas of 23- and 25-gauge vitrectomy systems. The infusion port is dis-inserted from the traditional infra-temporal microcannula and reaffixed in the supra-nasal microcannula. The surgeon, the operating microscope, and the foot pedals are then adjusted to a temporal orientation, and the instruments inserted through the temporally placed microcannulas.

Conclusions: The flexibility of interchangeable microcannulas in 23- and 25-gauge PPV systems permits intraoperative switching between superior and temporal surgical sites to better manage posterior segment pathology.
\end{abstract}

Keywords: Vitrectomy, intraoperative switch, vitreoretinal, transconjunctival, microcannula.

Adequate visualization and sufficient access to all vitreoretinal pathology is an important aspect of successful pars plana vitrectomy surgery, especially in the era of the more flexible, small-gauge, microincision, transconjunctival techniques. By utilizing the flexibility of interchangeable microcannulas in 23- and 25-gauge vitrectomy systems, a surgical method is described in which the infusion port is moved intraoperatively from the traditional infra-temporal location and placed supra-nasally, thus permitting a temporal surgical approach to better tackle superior and inferior vitreoretinal pathology.

\section{INTRODUCTION}

With significant advancement in techniques and refinements in surgical instrumentation, a trend of more frequent use and ever expanding indications for 23- and 25gauge microcannula-based pars plana vitrectomy (PPV) has emerged [1-10]. Compared to the traditional sutured 20gauge systems, 23- and 25-gauge vitrectomy permits the use of smaller, self-sealing, transconjunctival wounds. The advantages of such PPV techniques include: shorter operating time, less postoperative inflammation and patient discomfort, and more rapid visual recovery have been reported with both 23 - and 25-gauge PPV [1-6, 10, 11$]$.

*Address correspondence to this author at the Retina Service, Massachusetts Eye and Ear Infirmary, Harvard Medical School Department of Ophthalmology, 243 Charles St., Boston, Massachusetts 02114, USA; Tel: (617) 573-6874; Fax: (617) 573-3678;

E-mail: demetrios_vavvas@meei.harvard.edu
Additionally, 23- and 25-gauge systems have the added advantage of utilizing interchangeable mircocannulas for access into the eye $[1,11,12]$. With conventional 20-gauge PPV, the infusion cannula is traditionally sutured to the infra-temporal quadrant sclerotomy site. Two additional sclerotomy sites are then fashioned in the superior quadrants. In order to move the infusion port to a different sclerotomy site, the securing suture needs to be either cut or untied and another suture used to secure the infusion in place elsewhere. In contrast, with sutureless 23- and 25-gauge PPV, three identical microcannulas are employed for intraocular entry $[1,12]$. With the surgeon situated superiorly, operating from the head of the patient, the infusion port is typically attached to the infra-temporal microcannula. However, unlike the 20gauge infusion port that is sutured, with this interchangeable system, the infusion port can easily be removed from the microcannula and reinserted into any other microcannula in the eye. No further suturing is required and thus, the infusion port can be moved easily among any of the three microcannulas.

Despite these advantages, the 23- and 25-gauge systems also have several drawbacks. One major hindrance to wider adoption of 23- and 25-gauge surgery has been the perceived disadvantage of increased instrument flexibility when compared to 20-gauge systems [13]. Rotating the eye for adequate visualization and complete vitrectomy is more difficult due to this added instrument flexibility [1]. 23gauge instrumentation design has overcome some of the excessive flexibility of 25-gauge systems; however, eye 
maneuverability is still not equivalent to that seen with 20 gauge instruments [1]. While a majority of vitrectomies do not require the extreme maneuverability afforded by 20 gauge systems, in cases of superior retinal detachments, giant retinal tears and inferior retinal detachments in phakic patients, the surgical approach, even with the use of wideangle visualization lenses, becomes much more difficult.

In order to overcome the limitations imposed by instrument flexibility and to better visualize and approach vitreoretinal pathology in 23- and 25-gauge systems, the infusion port can be dis-inserted from the infra-temporal microcannula and attached to the supra-nasal microcannula. The surgery can then be carried out from the two temporal microcannulas with the surgeon sitting temporal to the patient.

\section{DESCRIPTION OF TECHNIQUE}

Preoperative eye preparation in all eyes includes eyelid and periorbital scrub with $10 \%$ povidone-iodine solution using standard sterile ophthalmic techniques. Furthermore, just prior to microcannula placement, the eye is flooded with an additional solution of $5 \%$ povidone-iodine. Three microcannulas (either 23- or 25-gauge) are inserted following generous displacement of both conjunctiva and Tenon's membrane as has been previously described $[1,5$, 11-13]. One microcannula is inserted infra-temporally, another supra-temporally and a third supra-nasally in the conventional fashion (Figs. 1, 2). A standard three-port PPV is started with the infusion port attached to the infratemporally placed microcannula. With the surgeon situated superiorly, the two superiorly placed microcannulas are utilized for intraocular vitrectomy instrumentation.

\section{Superior Approach Difficult Access}

When the location of the vitreoretinal pathology and/or the patient's anatomy prevents adequate visualization or surgical access and/or the instrument flexibility precludes sufficient maneuvering of the eyeball, a temporal approach to the vitrectomy may be employed (Figs. 1, 2). If no valve system is in place over the microcannula (such as that found in the Eckardt 23 Gauge Cannula System from DORC, Netherlands), a cannula plug is placed over the supratemporal microcannula. The infusion pressure is raised temporarily to $70 \mathrm{~mm} \mathrm{Hg}$ to maintain globe contour. (70 $\mathrm{mm}$ $\mathrm{Hg}$ was chosen in order to minimize the inevitable hypotony which occurs during the removal and then re-insertion of the infusion port.) The infusion port is then dis-inserted from the infra-temporal microcannula and reaffixed in the supra-nasal microcannula. Although it may be optional to increase the infusion pressure to $70 \mathrm{~mm} \mathrm{Hg}$, with the elevated infusion pressure, the re-insertion of the infusion port into the new microcannula is technically easier as the pressure inside the eye is better maintained with less hypotony. In over twodozen cases using this technique, we have encountered no complications (no retinal incarceration, nor intraocular hypotony, nor choroidal hemorrhages) from the switching of the infusion port. The surgeon, the operating microscope, and the foot pedals are then adjusted to a temporal orientation, and the instruments are now inserted through the temporally placed microcannulas (Figs. 1, 2). Vitreoretinal pathology which was previously inaccessible and challenging is now managed in a much more straightforward manner. The surgery can be completed from the temporal location or the infusion may be moved back to its original position depending on the surgical requirements. In certain surgical settings, it may even be advantageous to begin the

\section{Temporal Approach Improved Access}
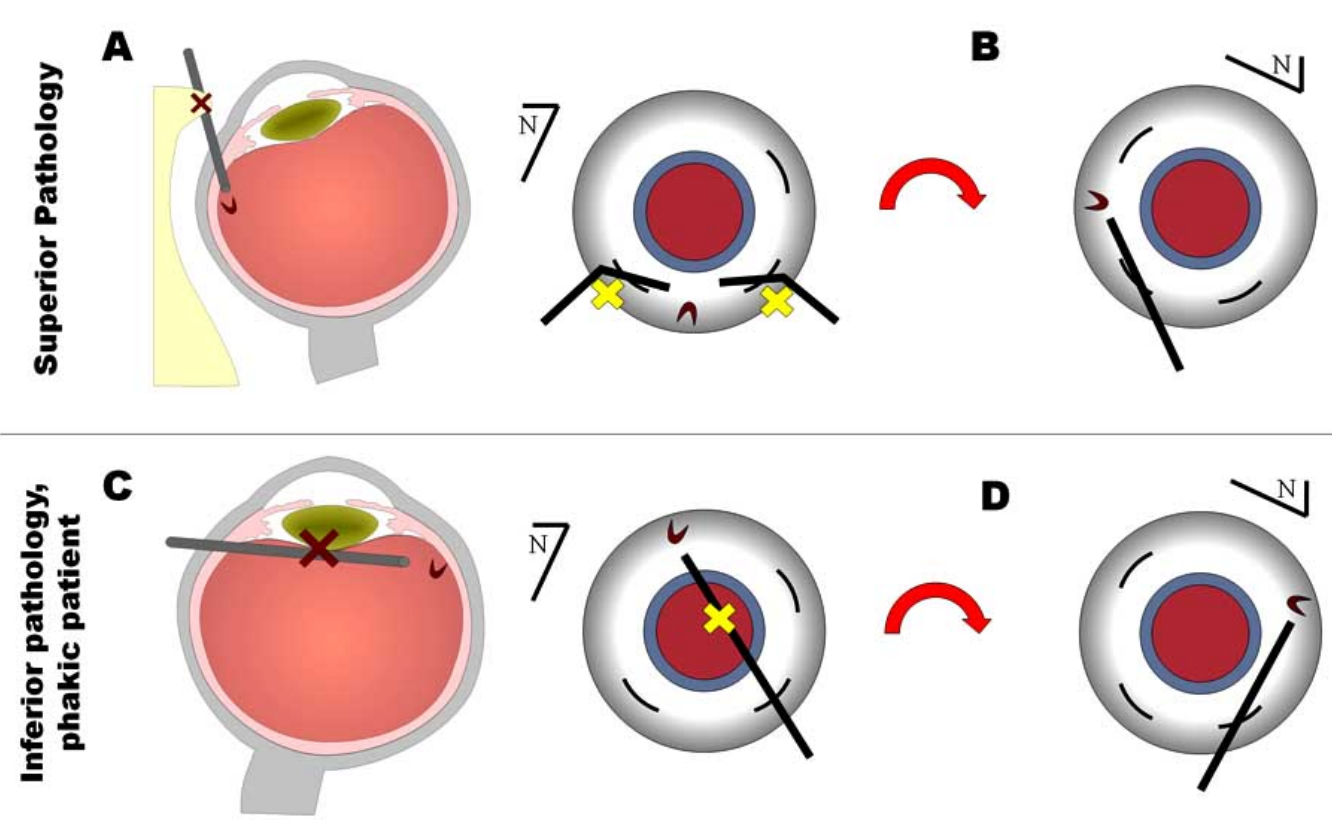

Fig. (1). (A) Superior vitreoretinal pathology or the patient's anatomy may prevent adequate visualization or surgical access and/or instrument flexibility may preclude sufficient maneuvering of the eye. (B) With a temporal surgical approach, vitreoretinal pathology, such as a superior retinal tear, may be more easily addressed. (C) Far inferior vitreoretinal pathology in a phakic patient may also be more difficult to tackle from a superior surgical position. (D) A temporal approach avoids potential lens complications. 

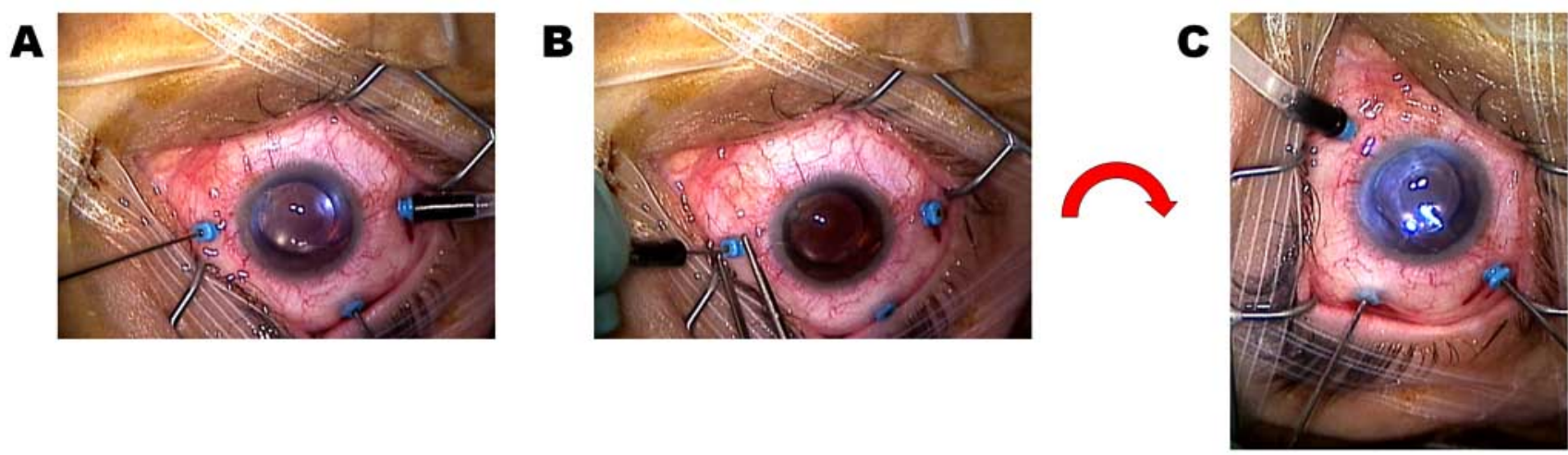

Fig. (2). The right eye of a patient undergoing 25-gauge microcannula-based transconjunctival pars plana vitrectomy (PPV). (A) The traditional approach to PPV with the infusion port placed infra-temporally and the surgeon situated superiorly operating via the two superiorly inserted microcannulas. (B) The infusion port is introduced into the supra-nasal microcannula following removal from its infratemporal location. (C) The surgeon, the operating microscope, and the foot pedals are adjusted to a temporal orientation, and the instruments are now inserted through the temporal microcannulas for the temporal approach to PPV.

vitrectomy from the temporal side, knowing that one can easily switch to a superior approach if necessary.

More recently, Lafetá et al. describe a 20-gauge transconjunctival sutureless vitrectomy trocar system [14]. This technique also offers the same flexibility in terms of the ability to change surgical position, with stiffer instrumentation when compared to 23- and 23-gauge systems.

\section{CONCLUSION}

A temporal approach in small-incision phacoemulsification for cataract extraction is a standard technique routinely utilized by anterior segment surgeons. However, such an approach is infrequently employed in the surgical management of posterior segment disease. The flexibility of interchangeable microcannulas in 23- and 25-gauge PPV systems (and even the newer 20-g microcannula based system) now allows vitreoretinal surgeons the option of intraoperatively switching between superior and temporal surgical sites to better manage posterior segment pathology. Unlike conventional non-trocar based vitrectomy techniques, microcannula-based systems do not require repeated suturing of the infusion port as it is placed from one part of the eye to another. In patients with anatomical limitations such as deep sockets and prominent nose bridge as well as in patients with superior retinal detachments, giant retinal tears and phakic inferior retinal detachments, a temporal approach to PPV may be more sensible and convenient than the customary superior approach.

\section{PATIENT CONSENT}

Consent for publication of figure had been obtained from the patient.

\section{REFERENCES}

[1] Williams GA. 25-, 23-, or 20-gauge instrumentation for vitreous surgery? Eye 2008; 22(10): 1263-66.

[2] Tewari A, Shah GK, Fang A. Visual outcomes with 23-gauge transconjunctival sutureless vitrectomy. Retina 2008; 28(2): 25862.

[3] Shah CP, Ho AC, Regillo CD, et al. Short-term outcomes of 25 gauge vitrectomy with silicone oil for repair of complicated retinal detachment. Retina 2008; 28(5): 723-8.

[4] Lai MM, Ruby AJ, Sarrafizadeh R, et al. Repair of primary rhegmatogenous retinal detachment using 25-gauge transconjunctival sutureless vitrectomy. Retina 2008; 28(5): 72934.

[5] Kim MJ, Park KH, Hwang JM, et al. The safety and efficacy of transconjunctival sutureless 23-gauge vitrectomy. Korean J Ophthalmol 2007; 21(4): 201-7.

[6] Rizzo S, Belting C, Cresti F, Genovesi-Ebert F. Sutureless 25gauge vitrectomy for idiopathic macular hole repair. Graefes Arch Clin Exp Ophthalmol 2007; 245(10): 1437-40.

[7] Riemann CD, Miller DM, Foster RE, Petersen MR. Outcomes of transconjunctival sutureless 25 -gauge vitrectomy with silicone oil infusion. Retina 2007; 27(3): 296-303.

[8] Miyahara T, Ohta K, Yamamoto Y, et al. 25-gauge vitrectomy to treat ocular complications of familial amyloid polyneuropathy. J Glaucoma 2007; 16(1): 169-70.

[9] Miura S, Ieki Y, Ogino K, Tanaka Y. Primary phacoemulsification and aspiration combined with 25 -gauge single-port vitrectomy for management of acute angle closure. Eur J Ophthalmol 2008; 18(3): 450-2.

[10] Shimada H, Nakashizuka H, Mori R, Mizutani Y. Expanded indications for 25-gauge transconjunctival vitrectomy. Jpn J Ophthalmol 2005; 49(5): 397-401.

[11] Chen E. 25-Gauge transconjunctival sutureless vitrectomy. Curr Opin Ophthalmol 2007; 18(3): 188-93.

[12] Fujii GY, De Juan E, Jr., Humayun MS, et al. A new 25-gauge instrument system for transconjunctival sutureless vitrectomy surgery. Ophthalmology 2002; 109(10): 1807-12.

[13] Kellner L, Wimpissinger B, Stolba U, et al. 25-gauge vs 20-gauge system for pars plana vitrectomy: a prospective randomised clinical trial. Br J Ophthalmol 2007; 91(7): 945-8.

[14] Lafetá AP and Claes C. Twenty-gauge transconjunctival sutureless vitrectomy tocar system. Retina 2007; 27(8): 1136-41. 